\title{
There is a Brown Dwarf Desert of Companions Orbiting Stars between 75 and 1000 AU
}

\author{
Chris McCarthy \\ Carnegie Institution of Washington/DTM 5241 Broad Branch Rd. NW \\ Washington D.C. 20015 \\ B. Zuckerman \& E. E. Becklin \\ Astronomy Division, University of California, Los Angeles, CA 90095
}

\begin{abstract}
We present results of the first large ( $>100$ stars) infrared coronographic search for substellar companions to nearby stars. The search consisted of two surveys of stars chosen for their youth and proximity to Earth: 1.) a 178 star infrared survey at Steward and Lick Observatories, with optical followup from Keck Observatory, capable of detecting companions with masses greater than $30 M_{J}$, orbiting between about 75 and $300 \mathrm{AU}, 2$.) a 102 star survey using the Keck telescope, capable of detecting extrasolar brown dwarfs and planets typically more massive than $10 M_{J}$, orbiting between about 75 and $300 \mathrm{AU}$.

This research resulted in the discovery of one brown dwarf companion, zero planets and 23 double stars. The frequency of brown dwarf companions (of any mass) to G, K and M stars orbiting between 75 and $300 \mathrm{AU}$ is measured to be $1 \pm 1 \%$. The frequency of massive $\left(>30 M_{J}\right)$ brown dwarf companions is found to be $0.6 \pm 0.6 \%$. The frequency of giant planets with masses larger than $10 M_{J}$, between 75 and $300 \mathrm{AU}$, is measured here for the first time to be no more than about $3 \%$.
\end{abstract}

\section{Introduction, Survey Results, \& Conclusion}

Precise radial velocity (PRV) searches sensitive to brown dwarf (BD) companions within $5 \mathrm{AU}$ have found very few $(\sim 0.3 \%$, Butler et al 2001$)$. The deficit of companions with $10<$ mass $<80 M_{J}$ distinguishes such objects from more numerous planets, and was referred to as the "brown dwarf desert" (BDD). The frequency of $\mathrm{BD}$ companions within $5 \mathrm{AU}$, is also deficient relative to the frequency of stellar companions. This is a second type of BDD.

To determine if the BDD (in the second sense) extends to greater radii, we obtained occulted J band images of 102 young stars using NIRC/Keck, and 178 young stars with Steward \& GEMINI/Lick. Candidates were screened for companionship using proper motion or optical colors obtained either from DSS or our own coronograph build for LRIS/Keck.

In spite of the above sensitivity limits, only $1( \pm 1)$ BD companion was detected, namely the binary proper motion companion to GL 577 (McCarthy, Zuckerman \& Becklin, 2001; Lowrance 2001), whose estimated individual masses 
straddle the substellar boundary. Using conservative estimates for the age distribution of our sample, and accounting for incompleteness, we measure BD companion fractions of: $0.6 \pm 0.6 \%$, for high mass $\left(>30 M_{J}\right)$ BDs between 120 and $1200 \mathrm{AU}$, and $1 \pm 1 \%$ for all BD's between 75 and $300 \mathrm{AU}$.

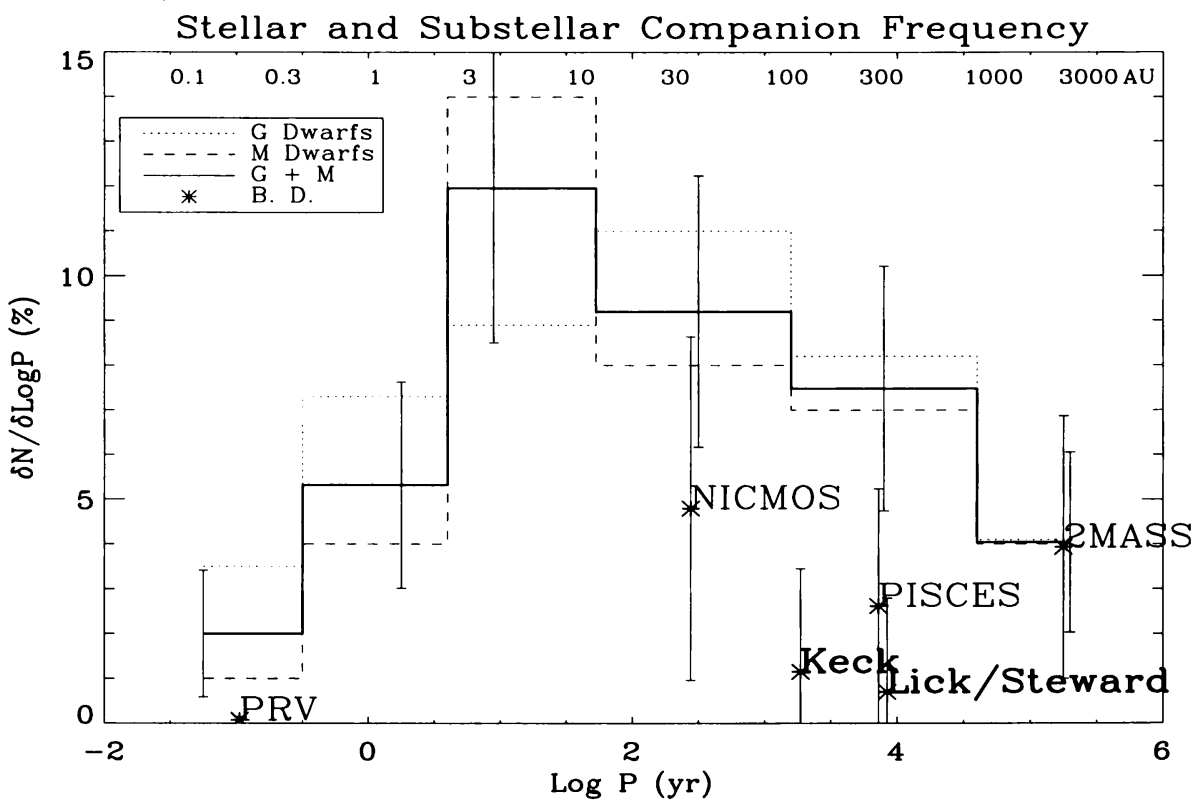

Fig. 1. We compare $6 \mathrm{BD}$ companion surveys, sensitive over a range of radii, by correcting for mass sensitivity by assuming a flat companion mass function: PRV (Butler et al), NICMOS (Lowrance), PISCES (Hinz et al) and 2MASS (Gizis, et al) These BD frequencies are compared with the frequencies of stellar companions, as determined by Duquennoy \& Mayor ( $G$ dwarfs) and Fischer \& Marcy (M dwarfs), in units of companions per unit log of orbital period in years, $d N / d \log P$, (Fischer \& Marcy Fig2b). For separations less than $1000 \mathrm{AU}$, there is a deficit of $B D$ companions relative to the stellar companion fractions. At separations greater than 1000 AU, BD companions appear to be just as rare as stellar companions.

\section{References}

Butler, R.P., et al 2001, in "Planetary Systems in the Universe", ASP Conference Series, Penny, A. , P. Artimowicz, A-M. Lagrange \& Russel, S. eds.

Duquennoy, A. \& Mayor, M, 1991, A\&A, 248, 485.

Fischer, D. \& Marcy,G., 1992. ApJ, 396,178

Gizis, J. 2001, ApJ, 551, 163.

Hinz, J. et al 2001 AJ, 123, 2027.

Lowrance, P. 2001. PhD Dissertation, U.C. Los Angeles.

McCarthy, C., 2001. PhD Dissertation, U. C. Los Angeles.

McCarthy, C., Zuckerman, B., \& Becklin, E., 2001, AJ, 121, 3259. 